\title{
Experimental Identification of Pilot Response Using Measured Data from a Flight Simulator
}

\author{
Jan Boril and Rudolf Jalovecky \\ Faculty of Military Technology, University of Defence, \\ Kounicova 65, 66210 Brno, Czech Republic \\ \{jan.boril, rudolf.jalovecky\} @unob.cz
}

\begin{abstract}
This paper describes the measuring of pilot response time to a sudden change in a controlled parameter whilst flying an aircraft. The authors of this paper created an analytical model of human behavior from the basic data of an automated regulation. The measurements have been done on a Cessna 152 simulator at the University of Hertfordshire, Hatfield. The tested pilots were pilot students with several tens of flight hours in real planes. The pilot's response to a sudden aircraft altitude change was measured. For analysis of the measured results a mathematical identification model in MATLAB ${ }^{\circledR}$ environment was used. The results obtained from MATLAB ${ }^{\circledR}$ confirm that the experimental measurements were successful.
\end{abstract}

Keywords: MATLAB ${ }^{\circ}$, Aircraft Control, Parameter Identification, Human Behavior Model, X-Plane, Pilot Response.

\section{Introduction}

In today's automated and digitalized world the stress is put on the development of both computers and artificial intelligence. However, a pilot or an operator is an indispensable part of any aircraft flying. Only time will tell if a pilot (operator) could be fully replaced by a machine and if so it will take a very long time. That's why the aircraft's manufactures started to do research on the influence of the human factor. The human factor influences many processes of aircraft flying from the very beginning of entering the cockpit, through taking off and landing procedure to stopping the engines. Taking the human efficiency into account the emphasis is put on the ergonomics of the controls in the cockpit, the manipulation space of the pilot, the method of entering the pre-flight data into the Flight Management System, autopilot controls, etc. These factors are supposed to make a pilot's work easy and eliminate his psychological and physical workload while flying a plane.

How will the pilot react in an unpredictable flight situation [1] if one of the automated systems were to cut off or if a sudden change of position angles would accrue due to weather conditions? The authors of this paper focused on weather conditions causing a sudden change of altitude or other flight parameters. Using experimental measurements from the flight simulator a model situation was created where the pilot's task was to react as fast as he could and put the aircraft back to the 
same altitude using only an elevator. The data from this flight simulator was analyzed. Only the most believable and the most interesting data was input into the MATLAB® environment with use an algorithm to identify parameters of a transfer function.

To determine the optimal mathematical model of a pilot's behavior when flying an aircraft is, from an automated control point of view, a very difficult and complex task. The reason is that the parameters and time constants of the pilot (as a human) are time variables and are influenced by many unpredictable factors such as the pilot's experience, tiredness, stress, surrounding noise etc. To determine a human's behavior, within a control loop, in a given flight mode is possible only after obtaining a correct pilot response in a given mode in the correct time. The authors identified, modeled and simulated these responses by measuring the pilot's responses in a flight simulator. From this data the best realistic time constants representing the pilot's behavior were found. The future vision of the authors is to set limits to all the pilot behavior time constants depending on the level of their experience and psychological and physical condition.

\section{Mathematical and Theoretical Background of the Experiment}

\subsection{Mathematical Model of a Pilot Behavior}

A human-pilot character in the control system can be represented by a variety of complex block diagrams which more or less describe most of the possible factors affecting human behavior [2,3]. Generally, it is not possible to create one universal model fully describing the human dynamic character in various situations during a flying process.

One possible model of human behavior dynamics is shown in the block diagram in Fig 1. It is very simplified, but very concise. There are 3 mutually interfering "blocks". The input - sensors are the pilot's sensory organs, from where the detected information goes into the central nervous system. The average speed of emotion transmission is in the range of 5 to $125 \mathrm{~ms}-1$. In an automated control system this transmission feature can be represented by a transport delay. The response time mainly depends on the level of the pilot's internal stress, the actual pilot's condition and perhaps also on some other factors. Sensory organ features are in real life represented by a sensitivity level, adaptation ability and the ability to mutually cooperate. After processing the received signal a command to hand or leg muscles is sent to adjust the elevator, aileron and rudder deflections. For maintaining the requested flight parameters the pilot uses three different types of regulators [3]:

- $\quad$ Predictive regulator, keeping the required flight mode based on the pilot's received visual and sensory perception of the flight.

- Feedback regulator, created by correct visual and sensory perception of the required flight mode.

- $\quad$ Precognitive regulator, recalling the learnt maneuver from memory, i.e. a clear sequence of elevator, aileron and rudder deflections making the required aircraft movement. 


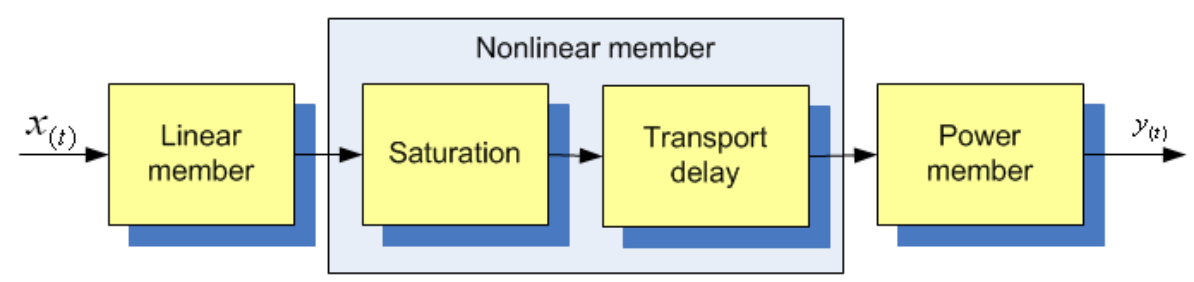

Fig. 1. One of the possible human behavior models in the machine

When analyzing any aircraft control with human behavior it is essential to take into account that all the human features are time variables and dependent on the actual pilot condition, psychological state, tiredness and ability to adapt to a new situation. This is all affected by long-term habits, education, training, etc. To create a mathematical model of a human in such a moment is not easy. For modeling human behavior a linear model is often being used (which is not quite correct for example regarding output value limitations) with a transport delay defined by a transfer function as follows $[4,5,6,7]$ :

$$
F_{(s)}=\frac{Y_{(s)}}{X_{(s)}}=K \frac{\left(T_{3} s+1\right)}{\left(T_{1} s+1\right)\left(T_{2} s+1\right)} e^{-\tau s},
$$

where:

$K$ - Pilot Gain - representing the pilot's ability to respond to an error in the magnitude of a controlled variable. Increasing of force on the steers in relation to their deflection (from 0.1 to 100 ).

$T_{1} \quad$ - Lag Time Constant - describes the ease with which the pilot generates the required input i.e. reaction ability to rate of change of input signal (0.1 to $0.4 \mathrm{~s})$

$T_{2}$ - Neuromuscular Lag Time Constant - represents the time constant associated with contraction of the muscles through which the control input is applied by the pilot. The dynamics properties of the pilot power member's components ( 0.05 to $0.2 \mathrm{~s})$.

$T_{3} \quad$ - Lead Time Constant - reflecting the pilot's ability to predict a control input (0 to $2 \mathrm{~s})$.

$\tau$ - Represents a pure time delay describing the period between the decision to change a control input and the change starting to occur (0.1 to $0.3 \mathrm{~s})$

This shape of the transfer function is based on the assumption and can be applied in cases where the pilot behaves as a linear member. In the real control loop to a certain extent non-linear elements are always take into account, as in the system pilot aircraft. In the literature [2], for cases where the nonlinearity of actuator is take into account can be found the extended shape of above mentioned transfer function in the shape: 


$$
F_{(s)}=\frac{Y_{(s)}}{X_{(s)}}=K \frac{\left(T_{3} s+1\right)}{\left(T_{1} s+1\right)\left(T_{2} s+1\right)} e^{-\tau s}+\text { remnant function, }
$$

The design of the remnant function is complicated procedure because it attempts to represent the non-linear component of pilot behavior. It is primary source is the pilot's ability to learn and adapt which results in non-linearity and non-steady behavior. The secondary contribution comes from such things as the experimental setup and experimentally injected noise that affect pilot response to other inputs. However, careful selection of the pilot model and task can help minimize remnant effect [2].

In fact the human operator does not perform controlling activities according to a linear model, but his control efforts are always loaded by negative effects of nonlinear elements such a hysteresis, dead zone, saturation or nonlinear variable gain. It is difficult to identify not only those elements but also include or placed elements into the regulation circuit which has multiple feedback.

\subsection{Algorithm for Experimental Identification of Transfer Function Parameters}

According to the selected type of transfer functions for the pilot model, it is possible for the time constants determination in human behavior models to use mathematical methods of the experimental identification of real systems.

If input and output signal and the approximate form of the transfer functions are known, is possible to use these methods to specify the parameters of the transfer function. A key advantage is the use of the simulation program MATLAB $®$, which already contains some functions for realization of necessary calculations.

Function "fminsearch" is looking for a minimum of scalar function of several variables. With its help the algorithm for experimental identification of transfer function parameters was assembled in the form:

$$
F_{e i}=\frac{a_{1} s+1}{b_{2} s^{2}+b_{1} s+1},
$$

with defined criterion condition

$$
f_{\min }=\sum\left(y_{i d}-y\right)^{2} .
$$

The above algorithm cannot manage calculation of the transport delay. Therefore, the program was completed with a simple subprogram to search the beginning of the pilot response (output value was not zero respectively bigger than the entered low value). After the transport delay evaluation for the identification algorithm the input pulse was moved to the response beginning. The results of the transport delay calculations at all types of pilots practically agree (with an accuracy of calculation step $0.01 \mathrm{~s}$ ) with input value. 


\section{Description of the Experimental Workplace and the Measuring Methods of Pilot Responses}

\subsection{Experimental Workplace}

The flight parameters and the generally measured values for transfer function parameter identification were measured during a three-month exchange program at the University of Hertfordshire, Hatfield. The university has a laboratory with flight simulators used for pilot training as well as for research purposes. The mentioned flight simulator is primarily intended for pilot's preparation especially for training flight procedures before flight, during and after the flight. The lab is under the auspices of a specialist in automated aircraft control. Our flight tests have been allowed only with good will of Dr. Rashid Ali. Based on his expert advice a Cockpit Simulator Cessna 152 was selected for our testing, see Fig. 2.

The flight simulator Cessna 152 consists of a Cessna 152 aircraft fuselage with two seats for crew. This fuselage is anchored to a static base fixed to the floor. The flight simulation was done by three projectors, projecting images onto a parabolic wall. Based on the research needs software X-Plane 9 from Laminar Research Company was used. The main advantage of this software is its precise and detailed simulation of flight physics for all individual aircrafts. The simulator as a whole is controlled by a PC - also called an Instructor Station. An instructor sitting at this station can change any flight parameters during the flight simulation. All control elements, flight instruments and control stick inside the cockpit are connected to the instructor station. The pilot can fully focus on flying the plane while the instructor can see all the real time parameters on his monitor.

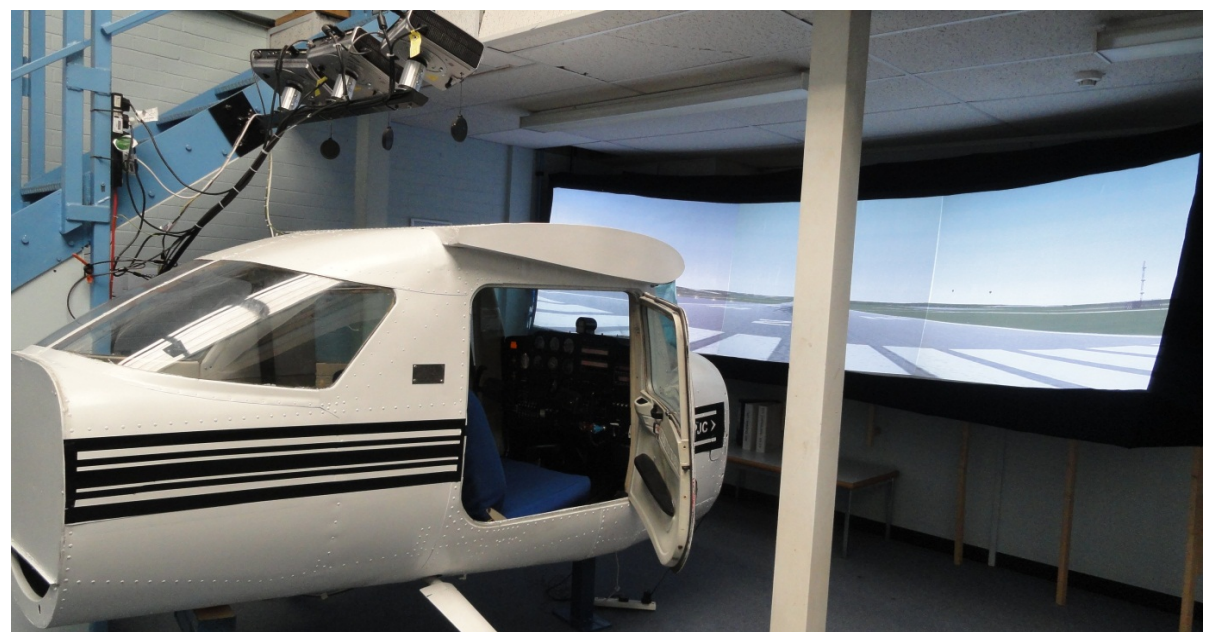

Fig. 2. Cessna 152 Cockpit Simulator (University of Hertfordshire) 


\subsection{Measurement Methodology of the Pilot Response}

The tested pilots were around the age of 23 and all holding Commercial Pilot Licenses (CPL). They were American university students on an exchange program at Hatfield, studying their final year of pilot studies. They all had several hours of flight experience on the Cessna 152 and Cessna 172 aircrafts. All tests were conducted in one day.

As the earlier created algorithm for transfer function parameter identification was made to process the input signal as a unit step function, the authors of this paper chose a unit step function (from a constant flight level) as an input signal. The test was conducted as follows. After an initial induction and simulator training the pilot was explained the test procedure and his task. The pilot's task was to take the plane into a straight horizontal flight. The instructor suddenly changed the aircraft altitude by 100 feet. In real situations such a decrease or increase in altitude can be caused by strong weather conditions or turbulences. The pilot's task was to put the aircraft back to the original altitude as fast as possible and stay there. He could do this by using only the aircraft elevator controls. The engine thrust was constant. The test was conducted with the same pilot several times in the same manner. Also the other pilots were tested in the same manner and under the same conditions. All of the data was recorded and stored in the instructor station.

\subsection{Factors Affecting the Measurements}

Some limiting factors, occurring during testing, affected the measured results. Firstly, in real situation the pilot senses any aircraft change by his senses organs. This cannot be ensured when using a simulator fixed to the floor. The tested pilots only sensed the altitude change visually by watching the altimeter in the cockpit and by expecting a sudden change. This fact largely influenced (increased) the time constant of the pilot transport delay between sensory perception of the change and a brain response.

After result evaluation and consultation with the pilots about the flight process the pilots talked about greater control sensitivity of the simulator compared to a real aircraft. Another factor lowering the realistic feel of the flight was a small observation angle as seen in Fig.2. Due to the distance and curvature of the screen used for image projecting the pilots didn't have $100 \%$ the same feeling as they would in a real aircraft cockpit.

\section{Simulation Result Analysis Using an Algorithm for Experimental Identification of Transfer Function Parameters}

The measurements from the simulator were analyzed using an algorithm for experimental identification of transfer function parameters. The authors have already created and tested such an algorithm. However, this was the first time realistically measured data from a simulator was applied. Four pilots were tested and each of them had to deal with four to six different changes of a flight altitude. The two cases below are the two best pilot's maneuvers, one going back up to the original altitude and one going back down to the original altitude. The last case demonstrates a badly conducted maneuver and the imperfection of the identification algorithm. 
Fig.3 shows an almost perfect pilot maneuver when returning to the original altitude. This was the pilot's fourth trial which proves that the more trials the better the pilot gets. The pilot was able to recover the original altitude in 14 seconds only by using an elevator control. Undoubtedly, the time in which the pilot is able to recover the original altitude also depends on the type of aircraft. The pilot's response is copying the graph of PID regulator to which the pilot can be compared. Taking in account a standard deviation, the pilot's response chart is almost perfect. To a person's naked eye, there is almost no difference between the pilot response curve and the curve created by the mathematical model for identification of transfer function parameters.
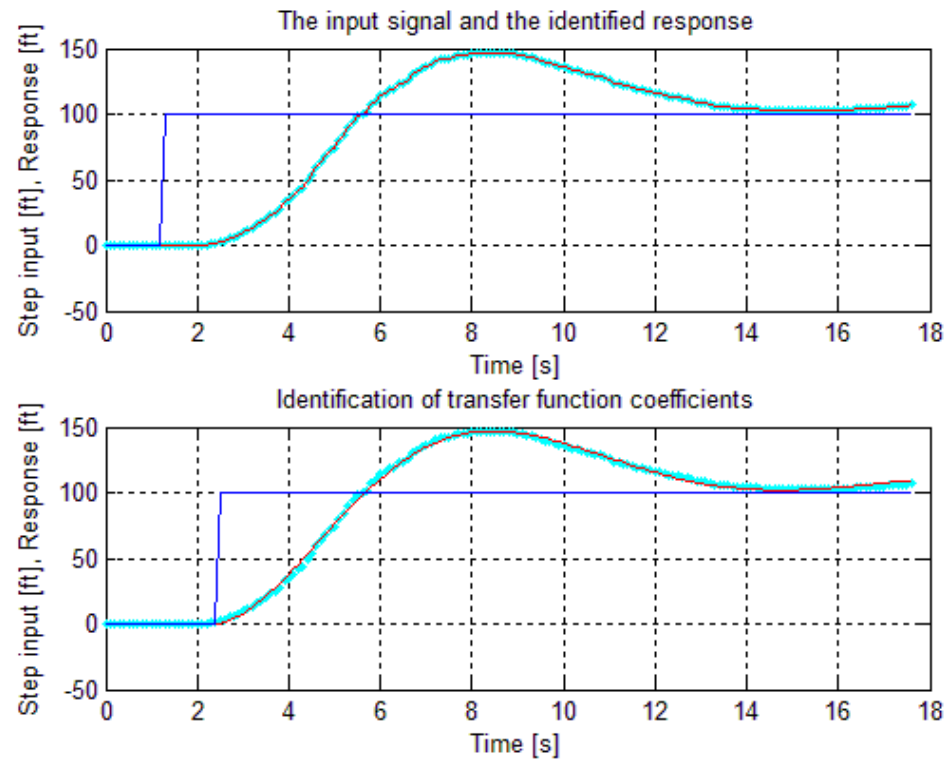

Fig. 3. Pilot's response to a unit step function - ascent to the original altitude

Fig.4 shows the best pilot's maneuver when descending to the original altitude. This result is absolutely unique. In the other tests none of the other pilots matched even slightly such a response curve. The identification algorithm approximated the pilot's response to the unit step function reasonably well. In Tab.1 important identification algorithm parameters are shown after several hundreds of iterations and also the pilot behavior time constants are shown, i.e. their product and their sum.

In Fig.5 there is clear evidence of the pilot's effort to come back to the original altitude. In this case the altitude recovery took longer and two aircraft oscillations occurred. Similar aircraft oscillations were found at least once for each pilot in their attempt to quickly descend back to the original altitude. The reason is that when descending the speed is naturally increasing and therefore the recovery maneuver is more difficult and the controls are more sensitive. It is also important to note that applying a $2^{\text {nd }}$ order transfer function for this case of pilot response was inadequate. All the simulation parameters and the pilot time constants from this analysis were disproportionally higher than those in the two cases mentioned above. An 
improvement could be reached by applying a higher order transfer function, but those results would not be comparable with the other measured results.
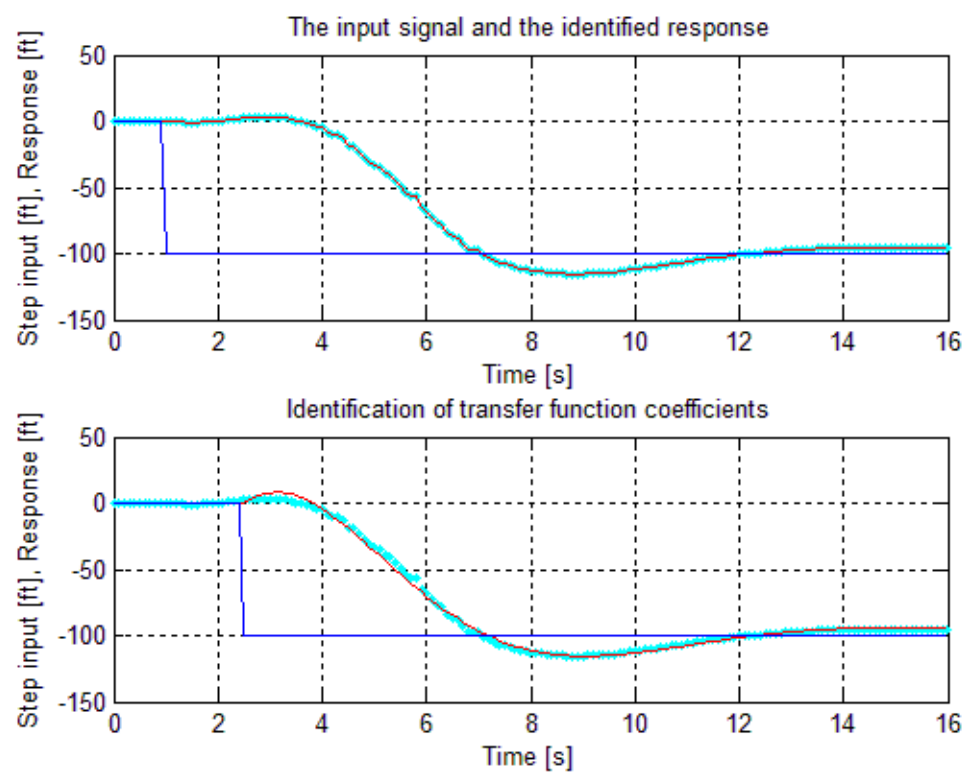

Fig. 4. Pilot's response to a unit step function - descent to the original altitude
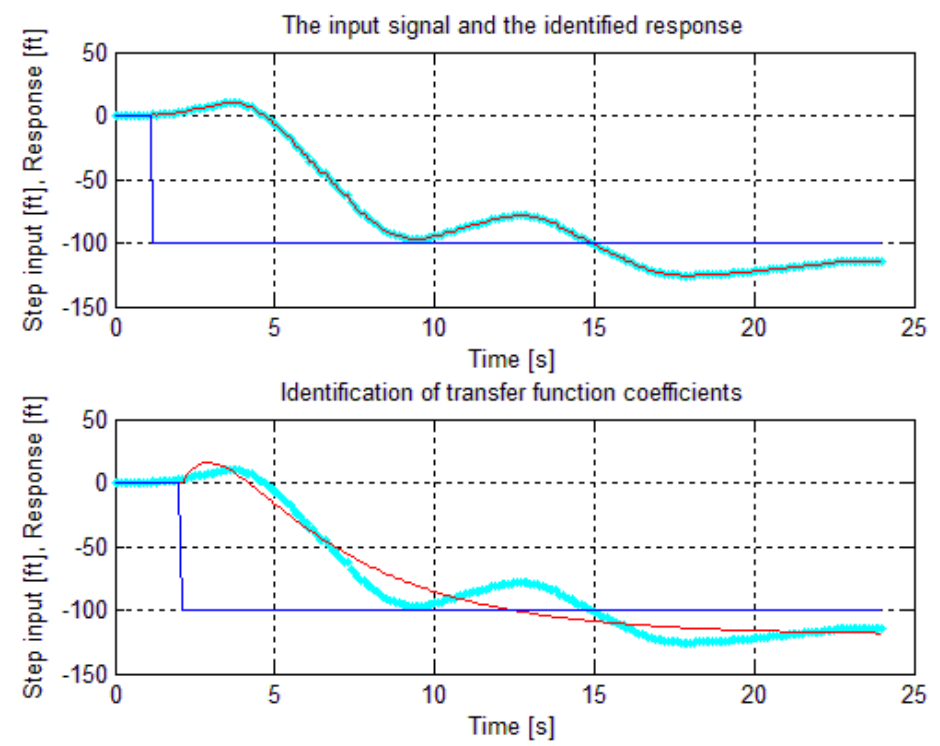

Fig. 5. Pilot's response to a unit step function - descent to the original altitude (oscillation) 
All the conducted tests show fairly higher time delays than assumed in theory [3]. That is caused by a wide range of factors affecting both the method of testing and assessment and the identification algorithm itself. There are two main reasons for these higher delays. Firstly, that the pilot was detecting the altitude change only visually. Secondly, the pilots were not informed when the altitude would suddenly drop or increase by the 100 feet and thus taking longer to analyze the situation and react appropriately. The authors also discovered, from the identified data, that aircraft dynamics also play an important role in getting a higher time delay. It is clear from the measured control stick responses that pilots started the returning maneuver about 0.2-0.3 sec earlier than the plane started to ascent or descent. The last but not least factor affecting the time delay is the sampling frequency set at $0.1 \mathrm{sec}$ disallowing more accurate time delay analyses.

Table 1. Parameters of the identified transfer functions

\begin{tabular}{ccccccc}
\hline Figure & Standard deviation & Proportional gain & Transport delay & $\mathrm{T}_{3}$ & $\mathrm{~T}_{1} \mathrm{~T}_{2}$ & $\mathrm{~T}_{1}+\mathrm{T}_{2}$ \\
\hline 3. & 1.56 & 1.12 & 1.1 & 0.28 & 3.46 & 1.32 \\
4. & 2.12 & 0.97 & 1.4 & -0.78 & 2.72 & 1.60 \\
5. & 10.33 & 1.20 & 0.8 & -1.58 & 4.11 & 5.30 \\
\hline
\end{tabular}

\section{Conclusion}

The authors of the paper conducted about twenty changes of a flight altitude on the Cessna 152 flight simulator from which they obtained a lot of data for parameter identification of a pilot behavior model transfer function. Only the most important and the most interesting pilot responses were chosen. When the measured time constants shown in Tab.1 were compared to the theoretical boundaries of the time constants it was clear that it is possible to simulate a realistic model of a pilot's behavior.

In the near future, the authors are planning to set up an aircraft simulator experimental laboratory in which testing and data assessment would continue to be improved. The algorithm for experimental identification will be refined so that the algorithm clearly separates the important pilot time constants needed for determining its limits.

The authors of this paper are aware that the last factor affecting their measurements is the pilot's simulator flight hours. This was also shown in the measurements where the pilots were getting better with each conducted trial. Their response time, in which they had to put the aircraft back into its original horizontal altitude, was shortening.

The above mentioned factor negatively affected both the pilot's lead time constant depending on his experience and lag time constant connected to his accustomed stereotype routine.

Acknowledgments. The paper was written under the umbrella of a project development department at the University of Defence - project K206 titled "Complex Electronic System for UAS" and supported by the association UDeMAG (University of Defence MATLAB Group). 


\section{References}

1. Boril, J., Jalovecky, R.: Response of the Mechatronic System, Pilot - Aircraft on Incurred Step Disturbance. In: 53rd International Symposium ELMAR 2011, pp. 261-264. ITG, Zagreb (2011)

2. McRuer, D.T., Krendel, E.S.: Mathematical Models of Human Pilot Behavior. AGARDAG-188 (1974)

3. Havlikova, M.: Diagnostic of Systems with a Human Operator, Doctoral Thesis, Brno University of Technology (2008) (in Czech)

4. Jalovecky, R., Janu, P.: Human - Pilot's Features During Aircraft Flight Control from Automatic Regulation Viewpoint. In: 4th International Symposium on Measurement, Analysis and Modeling of Human Functions, pp. 119-123. Czech Technical University in Prague, Czech Republic, Prague (2010)

5. Jalovecky, R.: Man in the Aircraft's Flight Control System. Advance in Military Technology - Journal of Science 4(1), 49-57 (2009)

6. Cameron, N., Thomson, D.G., Murray-Smith, D.J.: Pilot Modelling and Inverse Simulation for Initial Handling Qualities Assessment. The Aeronautical Journal 107(1744), 511-520 (2003)

7. Boril, J., Jalovecky, R.: Simulation of Mechatronic System Pilot - Aircraft - Oscillation Damper. In: ICMT 2011 - International Conference on Military Technologies, pp. 591597. University of Defence, Brno (2011) 